\title{
Análise Empírica de Comunidades Online baseadas em Enriquecimento Semântico para encontrar Usuários Especialistas
}

\author{
Thiago B. Procaci ${ }^{1}$, Bernardo Pereira Nunes ${ }^{1,2}$, \\ Fernando Pinhati ${ }^{1}$, Sean W. M. Siqueira ${ }^{1}$ \\ ${ }^{1}$ Programa de Pós-Graduação em Informática, Universidade Federal do Estado do Rio \\ de Janeiro (UNIRIO), Rio de Janeiro, RJ, Brasil \\ \{thiago.procaci, fernando.junior, sean\}@uniriotec.br \\ ${ }^{2}$ Departamento de Informática, PUC-Rio, Rio de Janeiro, RJ, Brasil \\ bnuneseinf.puc-rio.br
}

\begin{abstract}
Online communities have become important places for users to exchange information and build knowledge. In such communities, people ask or answer questions and learn with each other. However, some problems may occur such as getting no answer or getting contradictory ones. Looking for ways to minimize these problems, it would be important to identify people who are willing to help and who provide good answers (experts) in such communities. This work focus on finding experts in one online community using a semantic approach. We built an RDF graph and it was possible to find experts able to answer specific questions.
\end{abstract}

Resumo. Comunidades online possuem um papel importante na Web, promovendo a integração, colaboração e um ambiente informal de aprendizagem para seus usuários. Nessas comunidades, os participantes geralmente enviam e respondem perguntas, possibilitando aos usuários aprender uns com os outros. Contudo, nessas comunidades, alguns problemas podem ocorrer, como não obter respostas ou mesmo receber respostas erradas. Uma das alternativas para minimizar tais problemas é identificar especialistas dispostos a ajudar e que são capazes de fornecer boas respostas nessas comunidades. Este trabalho visa contribuir com a identificação de especialistas em comunidades online. Para isto, um estudo empírico baseado em tecnologias semânticas foi realizado para extração de conceitos em textos (perguntas e respostas). Um grafo RDF foi construído e, através de consultas, foi possível encontrar usuários com alta reputação para responder perguntas específicas.

\section{Introdução}

Devido às crescentes demandas por conhecimento dentro das organizações e uma disponibilidade limitada de recursos e competências para suprir tais demandas, muitos profissionais, tanto da indústria quanto da academia, acabam buscando por conhecimento em fontes externas para resolver os seus problemas [Constant et al., 1996], [Fritzen et al., 2013], [Prates et al., 2013], [West et al., 2014]. Essas fontes externas podem ser os motores de busca da Web, sites ou mesmo comunidades online. As comunidades online são compostas por indivíduos que compartilham interesses 


\section{CBIE-LACLO 2015}

Anais dos Workshops do IV Congresso Brasileiro de Informática na Educação (CBIE 2015)

comuns e voluntariamente trabalham para expandir a sua compreensão sobre um domínio do conhecimento [Alan et al, 2013], [Benson et al., 2012], [Marques et al., 2013] sendo, portanto, lugares apropriados para se procurar ajuda. Em geral, os membros dessas comunidades não se conhecem, podem ser identificados por pseudônimos e estão dispostos a ajudar uns aos outros por diversas razões: altruísmo, reputação, a reciprocidade esperada e os benefícios da aprendizagem [Kollock, 1999], [Lakhani \& Von Hippel, 2000], [Lin \& Huang, 2013]. O processo de postagem de perguntas e espera por respostas em uma comunidade online é denominada de consulta social (social query) [Banerjee \& Basu, 2008], [Morris et al., 2010], [Souza et al., 2013] e esta pode ser considerada uma alternativa mais qualitativa aos motores de busca. De acordo com Horowitz e Kamvar (2010), alguns problemas como perguntas mais contextualizadas, pedidos de recomendações, conselhos e opiniões são melhor resolvidos por humanos. No entanto, consultas sociais possuem algumas limitações. Por exemplo, quando perguntas são postadas, estas podem receber respostas erradas; usuários com dúvidas similares podem tentar modificar o contexto da pergunta; continuar recebendo respostas após a pergunta já ter sido resolvida; e, um dos pontos mais críticos, não receber resposta alguma [Morris et al., 2010], [Paul et al., 2011].

Considerando o cenário da aprendizagem informal encontrado nas comunidades online e buscando formas para minimizar algumas das limitações supracitadas das consultas sociais, este trabalho propõe uma abordagem para encontrar usuários que sejam mais adequados para responder a perguntas específicas, de um determinado domínio do conhecimento, postadas em comunidades online. Resumidamente, a abordagem proposta baseia-se na extração e reconhecimento de tópicos de perguntas e respostas através de tecnologias semânticas com o objetivo de identificar de perguntas e usuários candidatos capazes de responder a estas perguntas. Este trabalho está organizado da seguinte forma: a Seção 2 revisa trabalhos relacionados; a Seção 3 apresenta um estudo empírico em uma comunidade online visando caracterizá-las, bem como a proposta do trabalho. Por fim, a Seção 4 apresenta considerações finais e trabalhos futuros.

\section{Trabalhos Relacionados}

Estudos para encontrar usuários que dominam determinados assuntos (ou têm um conhecimento avançado), em uma comunidade online, já têm sido explorados na comunidade científica. Comumente, tais usuários são denominados como especialistas.

Neste contexto, existem trabalhos com foco em técnicas de recuperação de informações com processamento de linguagem natural (também conhecida como document-based) para identificar as competências de um usuário [Streeter \& Lochbaum, 1988] [Krulwich \& Burkey, 1996] [Ackerman \& Mcdonald, 1996]. Nessa abordagem, geralmente, os textos produzidos em ambientes virtuais são representados através de um vetor de termos (palavras ou tokens) com a sua respectiva frequência. Desta forma, é possível inferir qual o tipo de competência que cada usuário possui baseado em seus discursos. Todavia, o uso da abordagem com foco em recuperação de informação torna difícil elencar o nível de competência de cada usuário, uma vez que é difícil julgar se 


\section{CBIE-LACLO 2015}

Anais dos Workshops do IV Congresso Brasileiro de Informática na Educação (CBIE 2015)

um usuário fornece uma boa resposta somente fazendo uma tokenização ${ }^{1}$ de seus textos produzidos na comunidade e, em seguida, processando-os [Zhang et al., 2007].

Balog et al. (2009) propuseram uma forma para identificar os usuários especialistas baseada em consultas feitas em um ambiente e uma coleção de textos associados aos candidatos a especialistas. Este trabalho é baseado em técnicas de recuperação de informações e métodos probabilísticos e estatísticos visando determinar a relevância entre uma consulta e os candidatos a especialistas. Outro trabalho baseado em recuperação de informações e métodos probabilísticos foi apresentado por Liu et al. (2012), no qual foi proposto um framework que gerava automaticamente os perfis especializados dos usuários da comunidade. Esses perfis continham informações sobre as competências dos usuários e eram construídos baseados na associação entre os tópicos da comunidade com o perfil comum do usuário. Outra abordagem utilizada para isso é através de algoritmos de ranqueamento em grafos para encontrar os usuários especialistas de uma rede. A ideia dessa abordagem é aplicar algoritmos na comunidade (representada por um grafo), atribuindo um número para cada usuário simbolizando seu grau de competência em algum assunto. Campbell et al. (2003) e Dom et al. (2003) utilizaram o algoritmo de ranqueamento HITS em grafos para encontrar os usuários especialistas que faziam parte de uma lista de e-mail. Os resultados desses estudos foram animadores uma vez que a abordagem baseada em grafos se mostrou eficiente. Contudo, esses estudos tinham uma fraqueza: o tamanho da rede analisada. As redes eram relativamente pequenas e os resultados podiam não ser genéricos suficientes para refletir a realidade e serem aplicáveis a outros domínios. Zhang et al. (2007) propuseram a construção de um algoritmo baseado em grafos para o mesmo fim, porém, aplicado em um fórum de discussão online tradicional. Apesar da abordagem de Zhang et al. (2007) ter se mostrado interessante, os autores do trabalho concluíram, através de simulações, que comunidades com diferentes características devem ser analisadas separadamente, já que as características podem influenciar nos resultados obtidos, sendo necessárias adaptações nas medidas ou nas técnicas utilizadas. Alan et al. (2013) propuseram uma nova abordagem para identificar os usuários especialistas construindo um modelo híbrido da abordagem baseada em recuperação de informações como a baseada em algoritmos de ranqueamento em grafos.

Banerjee \& Basu (2008) apresentaram um algoritmo probabilístico que possibilitava direcionar perguntas para os usuários mais aptos a respondê-la. Esse algoritmo funcionava baseado em ações repetidas na rede no passado. Davitz et al. (2007) propuseram um trabalho similar, em que havia uma entidade global do sistema (agente) que monitorava a rede e determinava quais usuários receberiam (visualizariam) uma questão postada através de uma análise probabilística. Todavia, essa solução baseada em agentes foi testada somente em uma comunidade pequena. Souza et al. (2013) propuseram um algoritmo para encontrar os usuários especialistas que faziam parte da lista de seguidores de um usuário do Twitter. A ideia desse trabalho era encontrar o usuário seguidor com o perfil mais adequado para responder a uma pergunta no Twitter. Os resultados dessa pesquisa foram interessantes, pois o algoritmo proposto

\footnotetext{
${ }^{1} \mathrm{O}$ processo de tokenização pode ser considerado como a decomposição de um documento em termos que o compõe seguindo critérios específicos.
} 


\section{CBIE-LACLO 2015}

Anais dos Workshops do IV Congresso Brasileiro de Informática na Educação (CBIE 2015)

se mostrou eficaz para encontrar os usuários especialistas no Twitter. Contudo, a avaliação deste algoritmo foi feita com poucos usuários.

Procaci et al. (2014) apresentaram um estudo detalhado sobre métricas que podem ser evidências de confiabilidade de um usuário em cinco comunidades de perguntas e respostas. Neste trabalho, também foram estudadas técnicas baseadas em aprendizado de máquina para, em conjunto com as métricas, encontrar com precisão os usuários confiáveis. Contudo, foi possível somente identificar os usuários confiáveis de um domínio geral (como, por exemplo, especialistas em física). Dado o exposto, o presente trabalho tem como objetivo dar continuação ao estudo realizado em Procaci et al. (2014) de forma a possibilitar encontrar os usuários confiáveis com conhecimentos específicos dentro de um domínio. Isso se dará através do enriquecimento semântico dos textos produzidos pelos usuários em seus momentos de interação. Desta forma, se espera que especialistas possam ser facilmente encontrados para responder perguntas específicas dentro de uma comunidade online.

\section{Estudo Empírico}

\subsection{Dataset e Características Gerais das Comunidades}

Com a finalidade de realizar o estudo proposto neste trabalho foi escolhida uma comunidade de perguntas e respostas destinada ao aprendizado de Biologia denominada Biology Q\&A 2 (BQA). Para as análises foram extraídos: 2.317 usuários, 4.542 perguntas, 5.256 respostas, 7.381 comentários de perguntas e 7.070 comentários de respostas. Nessa comunidade, as discussões têm uma estrutura de trilhas (threads), ou seja, um usuário posta uma pergunta e, logo após, outros usuários postam respostas, comentários relativos à pergunta ou comentários relativos às respostas. Os comentários nessas comunidades são utilizados para clarificar algum ponto relativo às perguntas ou respostas. Cada usuário pode ser avaliado por outros usuários baseado em suas perguntas ou respostas postadas. Esse esquema de avaliação permite que os usuários construam a sua reputação na rede, podendo ser positiva ou negativa.

Estudos prévios mostram que é possível inferir a reputação de usuários dessas comunidades através de suas características [Procaci et al., 2014a], [Procaci et al., 2014b]. Em outras palavras, através dessas características é possível inferir se um usuário é especialista em um determinado domínio. Essas características podem ser: atributos ou métricas extraídas diretamente dos usuários como, por exemplo, o número de respostas que cada um forneceu; ou métricas derivadas através de representações abstratas, como grafos, que permitem extrair atributos como: o grau de entrada ou o resultado do algoritmo Page Rank que, no contexto da representação, podem ter um significado especial e indicar a reputação dos usuários. Esses estudos concluíram que, entre outras métricas, o número de respostas que um usuário fornece tem uma correlação forte com a reputação construída na comunidade. Isto é, quanto mais ativo um usuário é, maior tende a ser sua reputação e, portanto, mais confiável. Além disso, tais estudos relatam que, caso os usuários sejam agrupados com base em seus atributos, quanto maior for a média do número de respostas dos usuários dentro de cada grupo, maiores são as chances de encontrar usuários confiáveis.

\footnotetext{
${ }^{2}$ Site: http://biology.stackexchange.com/
} 


\section{CBIE-LACLO 2015}

Anais dos Workshops do IV Congresso Brasileiro de Informática na Educação (CBIE 2015)

\subsection{Enriquecimento Semântico}

Uma das formas para encontrar especialistas em determinado domínio é através de análises dos textos produzidos pelos usuários [Krulwich \& Burkey, 1996], [Ackerman $\&$ Mcdonald, 1996]. De forma diferente em relação às técnicas utilizadas nos trabalhos relacionados aqui descritos, nesta pesquisa foi utilizada a técnica de enriquecimento semântico para elencar os possíveis domínios de conhecimento de um usuário.

O enriquecimento semântico consiste na identificação de conceitos dentro de um texto. Por exemplo, na pergunta "O que é DNA?" pode-se extrair o conceito de DNA (que pertence ao domínio da biologia) que, por sua vez, pode ter conexões com outros conceitos [Nunes et al., 2014a] [Nunes et al., 2014b]. Neste cenário, foi utilizada a ferramenta DBpedia Spotlight ${ }^{3}$ para realizar o enriquecimento semântico dos textos produzidos pelos usuários nas comunidades de perguntas e respostas. Através desta ferramenta foi possível identificar conceitos relativos às perguntas, comentários de perguntas, respostas e comentários de respostas. Desta forma, se um usuário cita com determinada frequência um determinado conceito, isto, pode ser uma evidência que ele tem um interesse mais profundo neste assunto, dentro de um domínio [Franco et al., 2015). A partir desses conceitos identificados, foi construído um programa em Python para fazer a contagem de tais conceitos. A média de conceitos encontrados por pergunta na comunidade BQA foi 25 e por resposta foi de 39 .

\subsection{Capacidade para Responder Perguntas Específicas}

Suponha que uma nova pergunta específica seja postada em uma comunidade online. Quais são os usuários que possuem competência para respondê-la? A abordagem adotada nesta pesquisa é a da busca da relação existente entre os conceitos encontrados no texto de uma pergunta e os conceitos encontrados nos textos de suas respostas. Com a relação estabelecida, a ideia é identificar o usuário mais adequado para responder à questão. Neste trabalho, foi considerado como conceito de pergunta qualquer entidade presente no texto da pergunta, no título da pergunta e no comentário da pergunta. De forma similar, foi considerado conceito de resposta qualquer entidade encontrada no texto da resposta e no comentário da resposta.

O estudo dessa relação é importante, pois, se for verificado que os conceitos de uma pergunta estão presentes na resposta, este pode ser um bom indicador sobre o assunto específico que está sendo discutido [Streeter \& Lochbaum, 1988]. Logo, dada uma nova pergunta na comunidade, uma abordagem seria procurar por outra pergunta na comunidade que contém os mesmos conceitos (ou alguns) e indicar os usuários com maiores reputações que a responderam (ou usuários com o maior número de respostas fornecidas como demonstrado através de correlações em [Procaci et al., 2014]). Nesta lógica, seria possível encontrar os usuários com conhecimentos específicos para responder a nova pergunta postada. Como se pode perceber, de acordo com este trabalho, para encontrar os usuários especialistas são necessárias três etapas: (1) Identificar a relação entre conceitos de perguntas e conceitos de respostas; (2) Identificar quais conceitos são relevantes dentro da comunidade; (3) Buscar usuários com reputações significativas com base nos conceitos relevantes identificados.

\footnotetext{
${ }^{3}$ http://dbpedia-spotlight.github.io/demo/
} 


\subsection{Relação entre Conceitos da Pergunta e da Resposta}

A Tabela 1 mostra a relação entre conceitos das perguntas que estão presentes em alguma de suas respectivas respostas na comunidade analisada. Através da Tabela 1 é possível perceber que é relativamente fácil encontrar pelo menos um conceito de uma pergunta em alguma de suas respostas. Pelo menos $76,26 \%$ de todas as perguntas se encaixam neste caso. Esta verificação, apesar de apresentar uma porcentagem alta é frágil, pois, somente através de um conceito em comum entre uma pergunta e suas respostas, pode ser difícil identificar o assunto discutido. Na comunidade BQA, 12,43\% das perguntas possuem no mínimo $25 \%$ de seus conceitos em alguma de suas respostas. Aparentemente, a identificação de no mínimo $25 \%$ dos conceitos das perguntas em alguma de suas respostas é razoável para identificar assuntos em uma discussão. À medida que aumenta a quantidade mínima de conceitos que devem existir nas respostas, mais raros serão os casos, porém, possivelmente mais fácil será para identificar os tópicos comentados e o domínio dos usuários envolvidos.

Tabela 1. Relação entre Conceitos da Pergunta e Respostas - BQA

\begin{tabular}{|l|c|c|}
\hline \multicolumn{1}{|c|}{ Descrição } & Quantidade & Porcentagem \\
\hline Perguntas onde pelo menos um de seus conceitos está em alguma de suas respostas & 3.464 & $76,26 \%$ \\
\hline Perguntas onde nenhum de seus conceitos está em alguma de suas respostas & 1.078 & $23,74 \%$ \\
\hline Perguntas onde no mínimo 25\% de seus conceitos está em alguma de suas respostas & 565 & $12,43 \%$ \\
\hline Perguntas onde menos de 25\% de seus conceitos está em alguma de suas respostas & 3.977 & $87,57 \%$ \\
\hline Perguntas onde no mínimo 50\% de seus conceitos está em alguma de suas respostas & 167 & $3,67 \%$ \\
\hline Perguntas onde menos de 50\% de seus conceitos está em alguma de suas respostas & 4.375 & $96,33 \%$ \\
\hline Perguntas onde no mínimo 75\% de seus conceitos está em alguma de suas respostas & 77 & $1,69 \%$ \\
\hline Perguntas onde menos de 75\% de seus conceitos está em alguma de suas respostas & 4.465 & $98,31 \%$ \\
\hline Perguntas onde $100 \%$ de seus conceitos está em alguma de suas respostas & 35 & $0,77 \%$ \\
\hline Perguntas onde menos de $100 \%$ de seus conceitos está em alguma de suas respostas & 9.507 & $93 \%$ \\
\hline
\end{tabular}

\subsection{Grafo RDF}

Dada a relação entre conceitos das perguntas que também estão presentes nas respostas, foi criado um grafo RDF (Resource Description Framework) com base nas perguntas que possuem no mínimo $25 \%$ de seus conceitos em alguma de suas respostas. A justificativa do uso do grafo RDF está na necessidade de representação de entidades e suas relações. Além disso, futuramente, espera-se explorar relacionamentos entre as entidades desta pesquisa com as entidades de outros grafos RDF.

A ideia desse grafo reduzido é conseguir inferir quais assuntos um usuário discute, com base na relação de conceitos de perguntas e respostas. As entidades do grafo foram: "pergunta", "resposta", "conceito" e "usuário". Já os relacionamentos entre as entidades foram: "pergunta possui respostas", "usuário posta perguntas", "usuário posta respostas", "pergunta possui conceitos" e "resposta possui conceitos". Cada uma das entidades, além dos relacionamentos, possui um identificador e o usuário tem como atributo o número de respostas que ele postou na comunidade. Foram elencados 7 conceitos relevantes da Biologia para testar a proposta: DNA, vírus, espécies, fotossíntese, aves, código genético e evolução. A partir disso, uma consulta foi realizada nesse grafo com o objetivo de encontrar usuários com maiores chances de serem 
especialistas em um determinado conceito. A consulta, em alto nível, foi (ver Figura 1): retorne o usuário $U$ que respondeu à pergunta $\mathrm{R}$, e $\mathrm{R}$ possua o conceito $\mathrm{C}$, e o número total de respostas postadas por U na comunidade seja maior que a média do número de respostas postadas por outros usuários $U$ ' na comunidade, e U' também responderam $R$. Detalhando, o parâmetro da consulta será o conceito (por exemplo, DNA) e, a partir deste, a consulta deverá retornar o usuário correspondente. Note que, em nenhum momento na consulta, foi utilizada a reputação real para encontrar os usuários mais aptos para responder a uma pergunta. A ideia deste trabalho é usar o mecanismo de avaliação da comunidade, que permite construir a reputação (relatado na seção 3.1), somente como parâmetro para verificar a proposta. Ou seja, neste trabalho se busca uma forma automática para encontrar os usuários especialistas independentemente de qualquer mecanismo de avaliação de uma comunidade.

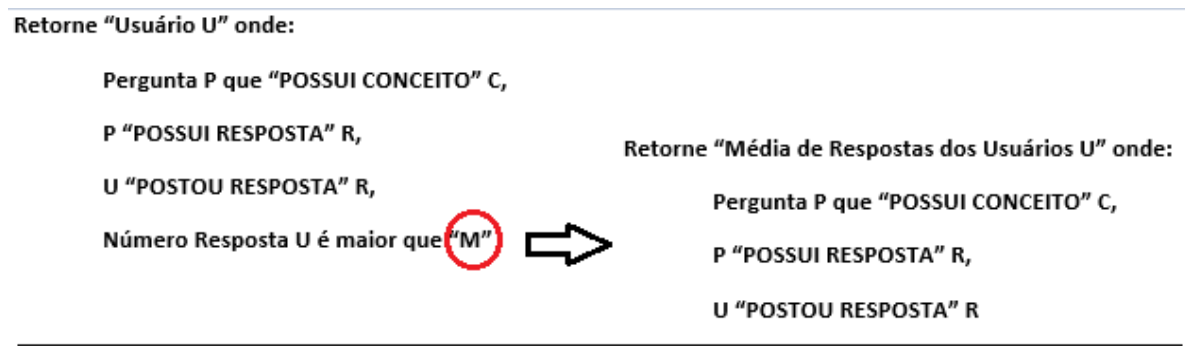

\footnotetext{
- A entidade usuário possui o atributo número de resposta que é a contagem de todas as respostas

fornecidas por ele na comunidade.

O parâmetro das consultas é o conceito (o mesmo para ambas).
}

Figura 1: Consulta

Tabela 2. Resultado Consultas no Grafo RDF

\begin{tabular}{|c|c|c|c|c|c|c|c|}
\hline $\begin{array}{l}\text { Conceito da } \\
\text { Consulta }\end{array}$ & $\begin{array}{l}\text { Menor } \\
\text { Rep. } \\
\text { Consulta }\end{array}$ & $\begin{array}{c}\text { Maior Rep. } \\
\text { Consulta }\end{array}$ & $\begin{array}{l}\text { Número } \\
\text { Usuário } \\
\text { Retornados }\end{array}$ & $\begin{array}{l}\text { Max. Rep. } \\
\text { Com. } \\
\text { (MAX) }\end{array}$ & $\begin{array}{l}\text { Média Rep. } \\
\text { Com. (M1) }\end{array}$ & $\begin{array}{c}\text { Média } \\
\text { Valores } \\
\text { entre M1 e } \\
\text { MAX (M2) }\end{array}$ & $\begin{array}{c}\text { Média } \\
\text { Valores } \\
\text { entre M2 e } \\
\text { MAX (M3) }\end{array}$ \\
\hline DNA & 2.886 & 16.659 & 27 & \multirow[t]{7}{*}{16.659} & \multirow[t]{7}{*}{193,47} & \multirow[t]{7}{*}{939,74} & \multirow[t]{7}{*}{$2.728,36$} \\
\hline Vírus & 2.886 & 16.659 & 8 & & & & \\
\hline Espécie & 2.757 & 16.659 & 18 & & & & \\
\hline Fotossíntese & 6.041 & 11.735 & 2 & & & & \\
\hline Aves & 5.280 & 11.735 & 2 & & & & \\
\hline Cód. Genético & 4.564 & 11.735 & 2 & & & & \\
\hline Evolução & 2.757 & 16.659 & 25 & & & & \\
\hline
\end{tabular}

A Tabela 2 mostra os resultados das execuções das consultas. Para fins de validação da consulta, foi efetuada uma comparação entre o usuário retornado e sua reputação adquirida ao longo do tempo na comunidade. Esta comparação pode ser visualizada na Tabela 2, que mostra a menor reputação de um usuário retornado (Menor Rep. Consulta) e o compara com o usuário de maior reputação (Max. Rep. Com.), com a média de reputação da comunidade (Média Rep. Com. M1). Além disso, duas outras médias são utilizadas para comparação: a M2, que é a média dos valores entre a máxima reputação e a reputação média da comunidade (M1); e a M3, que é a média dos valores entre a reputação máxima e a M2. A ideia disto é definir intervalos de proximidade de um determinado valor com o máximo da comunidade. Em outras palavras, os valores do 


\section{CBIE-LACLO 2015}

Anais dos Workshops do IV Congresso Brasileiro de Informática na Educação (CBIE 2015)

intervalo M3 são os mais próximos da reputação máxima. Os do intervalo M2 são menos próximos da reputação máxima quando comparados com M3 e mais próximos quando comparados com M1. Poderiam existir outros intervalos, contudo à medida que a reputação se aproxima da máxima, mais raros são eles. Através da Tabela 2, é possível perceber que, para cada consulta de um conceito, o usuário com menor reputação retornado (coluna "Menor Rep. Consulta") se encontra no intervalo de M3 (o mais próximo da reputação máxima). Isto significa que, a consulta realizada no grafo $\mathrm{RDF}$, construído utilizando $12,43 \%$ das perguntas da comunidade (bem como as respostas e usuários associados a essas perguntas), conseguiu encontrar usuários com alta reputação que provavelmente discutem assuntos relativos aos conceitos. Portanto, dada uma nova pergunta, uma abordagem para encontrar os especialistas seria identificar os conceitos presentes nela e realizar a consulta proposta.

\section{Conclusão}

Este trabalho apresentou um estudo para a identificação de usuários especialistas através de enriquecimento semântico em comunidades de perguntas e repostas online. Neste contexto, o presente trabalho utilizou como base de estudo uma comunidade online de Biologia. O estudo revela que $76,26 \%$ de todas as perguntas possuem pelo menos um conceito em comum com a resposta, ou seja, o processo de enriquecimento semântico foi capaz de encontrar conceitos em $76,26 \%$ das perguntas-respostas. Além disso, foi possível identificar que algumas mensagens possuíam no mínimo $25 \%$ dos conceitos descritos na pergunta em suas respostas. Esses números representam uma direção a seguir para encontrar usuários especialistas em domínios específicos. Dada a identificação de conceitos associados a um usuário, é possível sugerir ou mesmo filtrar usuários mais e menos prováveis para responder a uma nova pergunta postada numa comunidade online. Além disso, foi construído um grafo RDF baseado nas perguntas que possuem no mínimo $25 \%$ de seus conceitos em alguma de suas respostas. Em cima desse grafo reduzido (que representa só parte da comunidade), foram realizadas consultas para encontrar especialistas, dado um conceito. A consulta retornou resultados animadores, pois, todos os usuários retornados possuam uma boa reputação. Este estudo pretende fornecer base para novas pesquisas nessa área, utilizando ferramentas e tecnologias semânticas que possam dar suporte na identificação de usuários especialistas. Como trabalho futuro, planeja-se identificar mais conceitos chaves dentro da comunidade, associar os conceitos chaves aos usuários da comunidade e explorar as relações semânticas entre os conceitos para identificar com maior precisão especialistas.

\section{Referências}

Ackerman, M.S., McDonald, D.W, 1996. Answer Garden 2: merging organizational memory with collaborative help. In: Proceedings of CSCW '96, Boston, MA, ACM Press, 97-105.

Alan, W. G., Jian, J., Abrahams, A S., Fan, W., Zhang, Z., 2013. ExpertRank: A topicaware expert finding algorithm for online knowledge communities. In: Decision Support Systems, Volume 54, Issue 3. 1442-1451, ISSN 0167-9236, http://dx.doi.org/10.1016/j.dss.2012.12.020.

Balog, K., Azzopardi, L., Rijke, M. D., 2009. A language modeling framework for expert finding. In: Information Processing and Management, 45(1). 1-19. 


\section{CBIE-LACLO 2015}

Anais dos Workshops do IV Congresso Brasileiro de Informática na Educação (CBIE 2015)

Banerjee, A., Basu, S., 2008. A social query model for decentralized search. In: Proc. 2nd Workshop on Social Network Mining and Analysis, ACM Press.

Benson, V., Morgan, S., Tennakoon, H., 2012. A Framework for Knowledge Management in Higher Education Using Social Networking. In: International Journal of Knowledge Society Research (IJKSR), 3(2). 44-54. doi:10.4018/jksr.2012040104

Campbell, C.S., Maglio, P.P., Cozzi, A., Dom, B., 2003. Expertise identification using email communications. In: the twelfth international conference on Information and knowledge management, New Orleans, LA. 528-231.

Constant, D., Sproull, L., Kiesler, S., 1996. The kindness of strangers: the usefulness of electronic weak ties for technical advice. In: Organization Science 7 (2). 119.

Davitz, J., Yu, J., Basu, S., Gutelius D., Harris, A., 2007. iLink: search and routing in social networks. In: Proc. 13th ACM SIGKDD International Conference on Knowledge Discovery and Data Mining, ACM Press. pp. 931-940.

Dom, B., Eiron, I., Cozzi, A., Zhang, Y., 2003. Graph-based ranking algorithms for email expertise analysis. In: DMKD, New York, NY. ACM Press, 42-48.

Franco, A., Grimmer, J., Lee, M., Messing, S. 2015. Calling All Patriots: Communicating with our Representatives on Facebook. Disponível em: https://www.facebook.com/notes/facebook-data-science/calling-all-patriotscommunicating-with-our-representatives-on-facebook/10153231194653859

Fritzen, E., Siqueira, S. W. M., Andrade, L. C. V., 2013. Contextualized Search Enriched with Open Data for Supporting Collaborative Learning in Social Networks (Busca Contextualizada Enriquecida com Dados Abertos para Apoiar a Aprendizagem Colaborativa em Redes Sociais). In: Brazilian Journal on Computers and Education (Revista Brasileira de Informática na Educação), 21(3). 25-39.

Horowitz, D., Kamvar, S., 2010. The anatomy of a large-scale social search engine. In: Proc. of the 19th International Conference on World Wide Web (WWW), ACM Press. pp. 431-440.

Kollock, P., 1999. The economies of online cooperation: gifts and public goods in cyberspace. In: Smith, M.A. and Kollock, P. eds. Communities in Cyberspace, Routledge, London.

Krulwich, B., Burkey, C., 1996. ContactFinder agent: answering bulletin board questions with referrals. In: the 13th National Conference on Artificial Intelligence, Portland, OR. 10-15.

Lakhani, K., Von Hippel, E., 2000. How open source software works: "free" user-touser assistance. In: Research Policy, 32 (6). 923-943.

Lin, F., Huang, H., 2013. Why people share knowledge in virtual communities?: The use of Yahoo! Kimo Knowledge+ as an example. In: Internet Research, 23(2). 133159. http://dx.doi.org/10.1108/10662241311313295

Liu, X., Wang, G.A., Johri A., Zhou, M., Fan, W., 2012. Harnessing global expertise: a comparative study of expertise profiling methods for online communities. In: Information Systems Frontiers. 1-13. 


\section{CBIE-LACLO 2015}

Anais dos Workshops do IV Congresso Brasileiro de Informática na Educação (CBIE 2015)

Marques, A.M., Krejci, R., Siqueira, S.W.M., Pimentel, M., Braz, M.H.L.B., 2013. Structuring the discourse on social networks for learning: Case studies on blogs and microblogs. In: Computers in Human Behavior, 29(2). 395-400. http://dx.doi.org/10.1016/j.chb.2012.03.001.

Morris, M., Teevan, J., Panovich, K., 2010. What do people ask their social networks, and why?: a survey study of status message Q\&A behavior. In: Proc. 28th International Conference on Human Factors in Computing Systems (CHI), ACM Press. pp. 1739-1748.

Nunes, B.P., Kawase, R., Fetahu, B., Casanova, M.A., de Campos, G.H.B., 2014a. Educational Forums at a Glance: Topic Extraction and Selection. In: 15th International Conference on Web Information Systems Engineering (WISE), 2014. pp. 351-364.

Nunes, B.P., Mera, A., Kawase, R., Fetahu, B., Casanova, M.A., de Campos, G.H.B., 2014b. A Topic Extraction Process for Online Forums. In: IEEE 14th International Conference on Advanced Learning Technologies (ICALT), 2014. pp. 541-543.

Paul, S., Hong, L., Chi, E., 2011. Is twitter a good place for asking questions? a characterization study. In: The Eighth International Conference on Internet and Web Applications and Services Conference on Weblogs and Social Media (ICWSM). pp. 578-581.

Prates, J.C., Fritzen, E., Siqueira, S.W.M., Braz, M.H.L.B., Andrade, L.C.V., 2013. Contextual web searches in Facebook using learning materials and discussion messages. In: Computers in Human Behavior, 29(2). 386-394. http://dx.doi.org/10.1016/j.chb.2012.06.002.

Procaci, T. B., Siqueira, S. W. M., Braz, M.H.L.B., Andrade, L. C. V. 2014. How to find people who can help to answer a question? - Analyses of metrics and machine learning in online communities. Computers in Human Behavior, v. 47, p. 1.

Procaci, T. B., Siqueira, S. W. M., Andrade, L. C. V. 2014b. Finding Reliable People in Online Communities of Questions and Answers - Analysis of Metrics and Scope Reduction. In: 16th International Conference on Enterprise Information Systems, Lisbon. Proceedings of the 16th International Conference on Enterprise Information Systems. v. 16. p. 526-535.

Souza, C. C., Magalhães, J. J., Costa, E. B., Fechine, J. M., 2013. Social Query: A Query Routing System for Twitter. In: The Eighth International Conference on Internet and Web Applications and Services (ICIW).

Streeter, L., Lochbaum, K., 1998 Who Knows: A System Based on Automatic Representation of Semantic Structure. In: Proceedings of RIAO. 380-388.

West, J., Salter, A., Vanhaverbeke, W., Chesbrough, H., 2014. Open innovation: The next decade. In: Research Policy, 43(5). 805-811.

Zhang, J., Ackerman, M.S, Adamic, L., 2007. Expertise networks in online communities: structure and algorithms, In: Proceedings of the 16th international conference on World Wide Web, May 08-12, Banff, Alberta, Canada. 